\title{
Gestational Breast Cancer - MISCELLANEOUS PRESENTATIONS WITH CONSISTENT POOR OUTCOMES
}

\author{
Morais Rodrigues, l.; Nobre Pinto, A.; Riscado, I.; Veríssimo, C. \\ Obstetrics/Gynecology, Hospital Beatriz Ângelo, Loures, Lisbon, Portugal
}

$\checkmark$ Gestational breast cancer is defined as breast cancer diagnosed during pregnancy, first postpartum year and, for some authors, any time during lactation.

$\checkmark$ It is one of the most common cancers in pregnant women, affecting 15-35/100 000 deliveries.

$\checkmark$ There are several possible presentations of breast cancer affecting woman during and after pregnancy.

$\checkmark$ Delays in diagnosis are frequent; it is frequently at an advanced stage when diagnosed.

Descriptive study - clinical record analysis of the three cases diagnosed and surveilled in the last two years, in Hospital Beatriz Ângelo, a portuguese public hospital.

$\rightarrow 2500$ deliveries/year

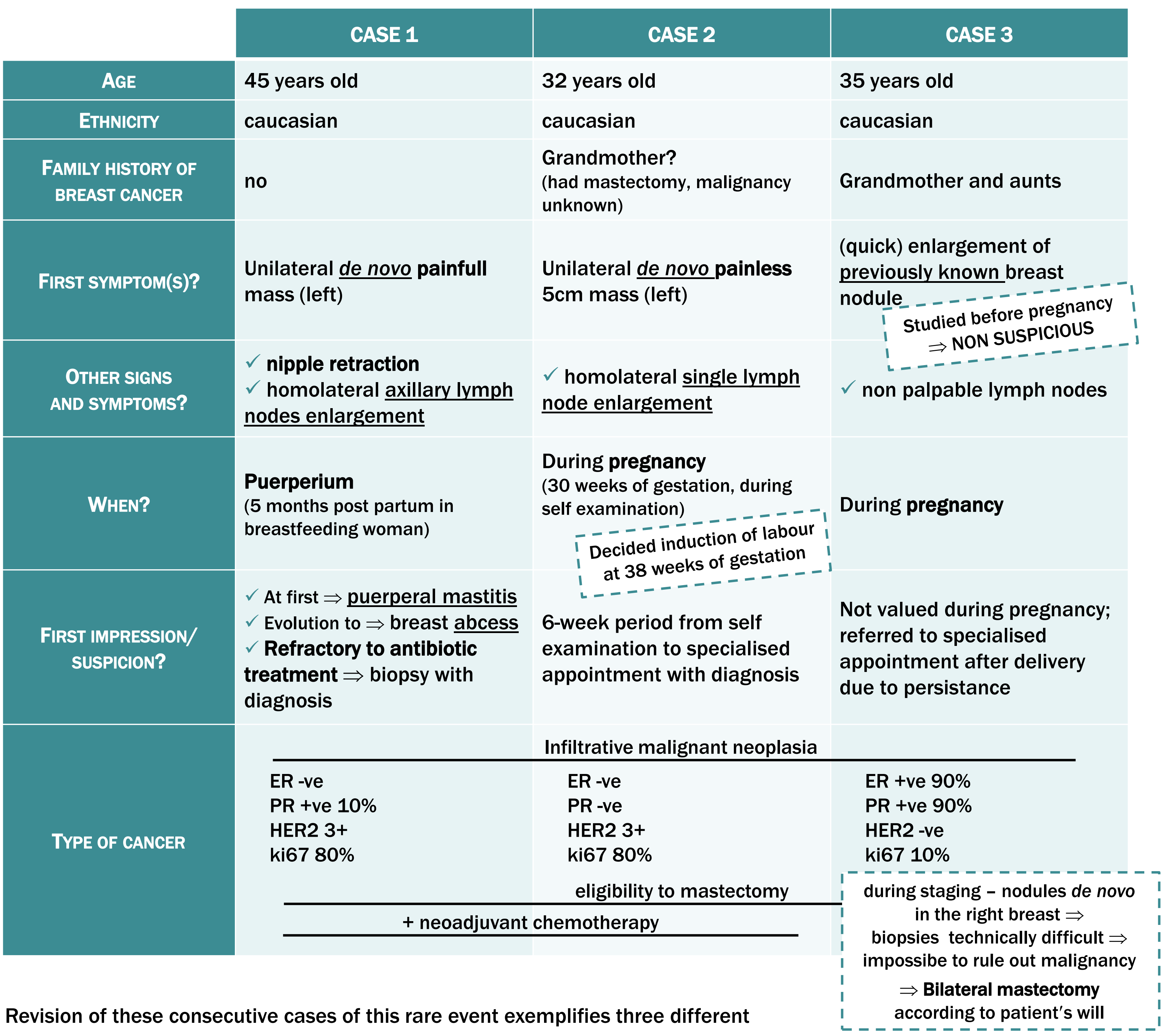
presentations, all diagnosed at an advanced stage

$\checkmark$ Awareness to this entity is essential to allow early diagnosis and treatment 